
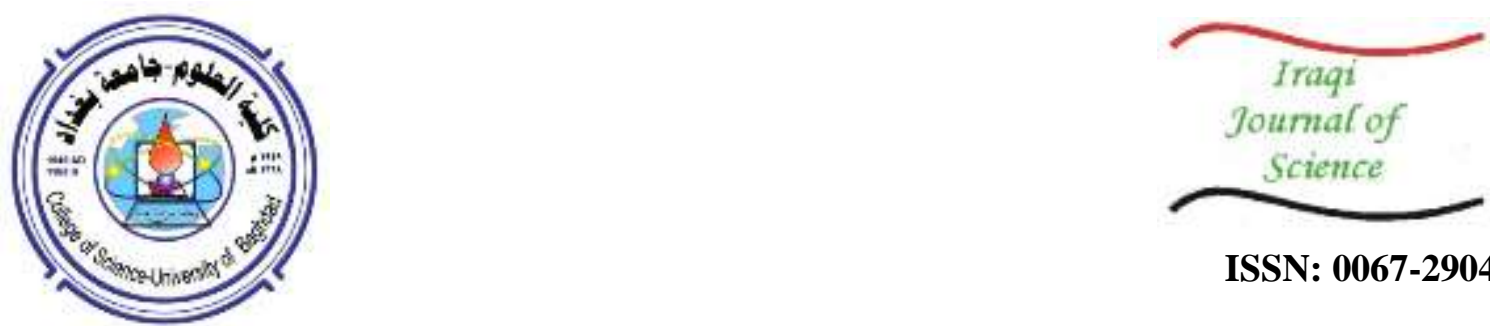

ISSN: 0067-2904

\title{
Laplace Adomian and Laplace Modified Adomian Decomposition Methods for Solving Nonlinear Integro-Fractional Differential Equations of the Volterra-Hammerstein Type
}

\author{
Shazad Sh. Ahmed*', Shokhan Ahmed Hama Salih', Mariwan R. Ahmed ${ }^{2}$ \\ ${ }^{1}$ Department of Mathematics, College of Science, Sulaimani University, Sulaimanyah, Kurdistan Region, Iraq. \\ ${ }^{2}$ Department of Science, College of Basic Education, Charmo University, Chamchamal, Kurdistan Region, Iraq.
}

\begin{abstract}
In this work, we will combine the Laplace transform method with the Adomian decomposition method and modified Adomian decomposition method for semianalytic treatments of the nonlinear integro-fractional differential equations of the Volterra-Hammerstein type with difference kernel and such a problem which the kernel has a first order simple degenerate kind which the higher-multi fractional derivative is described in the Caputo sense. In these methods, the solution of a functional equation is considered as the sum of infinite series of components after applying the inverse of Laplace transformation usually converging to the solution, where a closed form solution is not obtainable, a truncated number of terms is usually used for numerical purposes. Finally, examples are prepared to illustrate these considerations.
\end{abstract}

Keywords: Integro-Fractional Differential Equation, Caputo-fractional derivative, Adomian decomposition method, Modified Adomian decomposition method, Noise term phenomenon, Laplace transform.
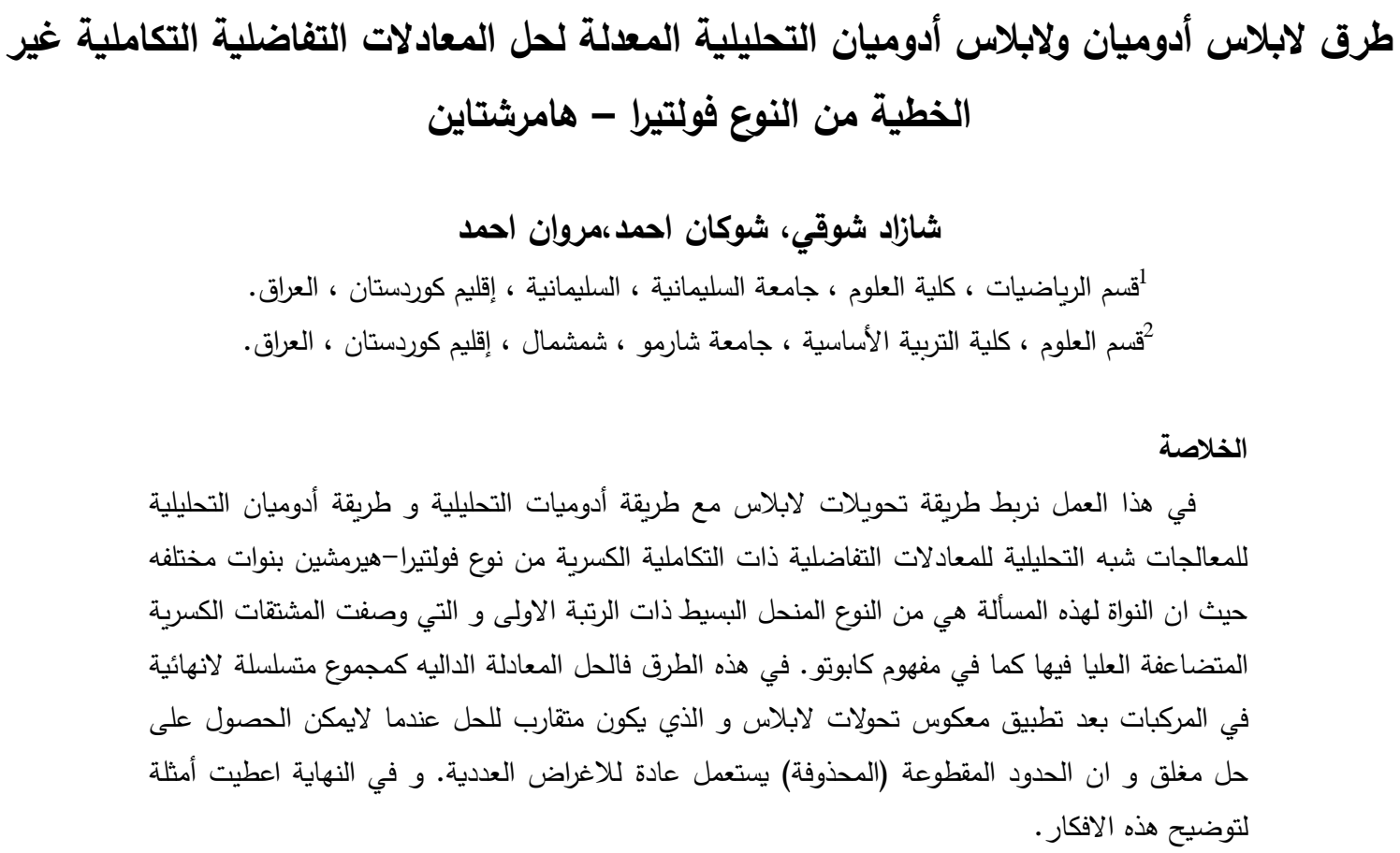


\section{Introduction:}

The goal of this work is to provide a semi-analytic solution for treating approximately the multihigher different fractional orders of nonlinear Integro-Differential Equations (IFDE) for VolterraHammerstein $(\mathrm{V}-\mathrm{H})$ type with the constant coefficients in the general form:

$$
\begin{aligned}
{ }_{0}^{C} D_{t}^{\alpha_{n}} u(t)+\sum_{i=1}^{n-1} \mathcal{C}_{i}{ }_{0}^{C} D_{t}^{\alpha_{i}} u(t)+\mathcal{C}_{0} u(t) \\
\quad=f(t)+\sum_{\ell=0}^{m} \lambda_{\ell} \int_{0}^{t} \mathcal{K}_{\ell}(t, s) \mathcal{H}_{\ell}\left(s,{ }_{0}^{C} D_{s}^{\beta_{\ell}} u(s)\right) d s, t \in[0, \mathcal{T}]
\end{aligned}
$$

with the initial conditions

$$
u^{k}(0)=u_{k} \in \mathbb{R} ; k=0,1, \cdots, \mu-1 ; \mu=\max \left\{\left[\alpha_{n}\right],\left[\beta_{m}\right\rceil\right\} .
$$

Where $u(t)$ is the unknown function which is the solution of equation (1) under initial condition (2), as well as, the functions $\mathcal{K}_{\ell}: S \rightarrow \mathbb{R}$ with $\left(S=\{(t, s): 0 \leq s \leq t \leq \mathcal{T}\} ; \mathcal{H}_{\ell}: S_{*} \times \mathbb{R} \rightarrow\right.$ $\mathbb{R}\left(S_{*}=\{s: 0 \leq s \leq t ; t \leq \mathcal{T}\}\right) ; \ell=0,1, \cdots, m$, and $f:[0, \mathcal{T}] \rightarrow \mathbb{R}$ is a continuous function. In addition, $\alpha_{i}, \beta_{\ell} \in \mathbb{R}^{+}, \ell=1,2, \cdots, m$ and $i=1,2, \cdots, n$ with property that $\alpha_{n}>\alpha_{n-1}>\cdots>\alpha_{1}>$ $\alpha_{0}=0, \beta_{m}>\beta_{m-1}>\cdots>\beta_{1}>\beta_{0}=0$ and $\lambda_{\ell}$ (for all $\ell$ ) are the scalar parameters. ${ }_{0}^{C} D_{t}^{\gamma}$ Denotes the Caputo fractional derivative of order $\gamma \in \mathbb{R}^{+} ;\left(\gamma=\alpha_{i(=\overline{1: n})}\right.$ or $\left.\beta_{\ell(=\overline{1: m})}\right)$.

Many mathematical modeling of various physical phenomena contains nonlinear fractional order Volterra integro differential equations, such as heat conduction in materials with memory [1]. Moreover, these equations are encountered in combined conduction, convection, radiation problems, fluid dynamics, biological models and chemical kinetics [1,2,3].

Adomian decomposition method and Laplace operator are widely used by many authors and researches to solve the problems in applied sciences such as differential, fractional differential, integral and integro-differential equations, see Ref $[4,5,6,7,8]$. In this work, we extend this technique to further deal with considering problem (1) with initial conditions (2).

The paper is organized as follows. Section 2 presents the necessary definitions and basic preliminaries of the fractional calculus, Adomian decomposition method and the Laplace transform; section 3 devotes to formulation of ADM and MADM with the aid of Laplace transforms for solving nonlinear IFDE for difference and simple degenerate kernels of V-H type; our results illustrated throughout examples in section 4. Finally, section 5 includes a discussion for these methods.

\section{Preliminaries and Notations:}

In this section, we discuss some basic definitions and properties of the fractional calculus theory and the Adomian decomposition method with Laplace transformations which are used further in this paper.

\subsection{Fractional Calculus:}

Fractional calculus deals with the differentiation and integration of arbitrary order, many definitions of it have been proposed [9,10,11]. Most frequently occurring are Riemann-Liouville and Caputo $[4,8]$. In this section, we discuss the definition and important properties of these two types of fractional derivatives, which are used throughout this paper. Moreover, some formulas which enjoy with a fractional operator with Laplace transformation. we begin by defining the function space $C_{\gamma}, \gamma \in \mathbb{R}$, which was used in development of the operational calculus for the differential operator.

Definition 1: [12]

A real valued function $u$ defined on $[a, b]$ be in the space $C_{\gamma}[a, b], \gamma \in \mathbb{R}$, if there exist a real number $p>\gamma$, such that $u(t)=(t-a)^{p} u_{*}(t)$, where $u_{*} \in C[a, b]$, and it is said to be in the space $C_{\gamma}^{n}[a, b]$ if and only if $u^{(n)} \in C_{\gamma}[a, b], n \in \mathbb{N}_{0}$.

Definition 2: $[10,13]$

Let $u \in C_{\gamma}[a, b], \gamma \geq-1$ and $\alpha \in \mathbb{R}^{+}$. Then the Riemann-Liouville fractional integral operator ${ }_{a} J_{t}^{\alpha}$ of order $\alpha$ of a function $u$, for $\alpha>0$ is defined by:

$$
{ }_{a} J_{t}^{\alpha} u(t)=\int_{a}^{t} \frac{(t-\xi)^{\alpha-1} u(\xi)}{\Gamma(\alpha)} d \xi, \quad \alpha \geq 0
$$

and for $\alpha=0$, we have ${ }_{a} J_{t}^{0} u(t)=I u(t)=u(t)$. 
Definition 3: $[10,13]$

The Caputo fractional derivative operator ${ }_{a}^{C} D_{t}^{\alpha}$ of order $\alpha \in \mathbb{R}^{+}$of a function $u \in C_{-1}^{m}[a, b]$ and $m-1<\alpha \leq m, m \in \mathbb{N}$ is defined as:

$$
{ }_{a}^{C} D_{t}^{\alpha} u(t)={ }_{a} J_{t}^{m-\alpha} D_{t}^{m} u(t)
$$

Thus for $\alpha=m\left(\in \mathbb{N}_{0}\right)$, and $u \in C^{m}[a, b]$, we have for all $a \leq t \leq b$

Note that: $[9,10,11,13]$

$$
{ }_{a}^{C} D_{t}^{0} u(t)=u(t) ;{ }_{a}^{C} D_{t}^{m} u(t)=D_{t}^{m} u(t)=\frac{d^{m} u(t)}{d t^{m}}
$$

i. For $\alpha \geq 0$ and $\beta>0$, then $J_{t}^{\alpha}\left\{(t-a)^{\beta-1}\right\}=\frac{\Gamma(\beta)}{\Gamma(\beta+\alpha)}(t-a)^{\beta+\alpha-1}$.

ii. For all $\alpha \geq 0, \beta \geq 0$ and $u(t) \in C_{\gamma}[a, b], \gamma \geq-1$, then:

$$
{ }_{a} J_{t}^{\alpha}{ }_{a} J_{t}^{\beta} u(t)={ }_{a} J_{t}^{\beta}{ }_{a} J_{t}^{\alpha} u(t)={ }_{a} J_{t}^{\alpha+\beta} u(t)
$$

iii. The Caputo derivative of any constant function is zero, i.e., ${ }_{a}^{C} D_{t}^{\alpha} \mathcal{C}=0 ; \mathcal{C}$ is any constant and $\alpha$ is arbitrary positive real numbers.

iv. Assume that $u \in C_{-1}^{m}[a, b] ; \alpha \geq 0, \alpha \notin \mathbb{N}$ and $m=\lceil\alpha\rceil$ then ${ }_{a}^{c} D_{t}^{\alpha} u(t)$ is continuous on $[a, b]$, and $\left[{ }_{a}^{C} D_{t}^{\alpha} u(t)\right]_{t=a}=0$.

v. Let $\alpha \geq 0, m=\lceil\alpha\rceil$ and $u \in C^{m}[a, b]$, then, the relation between the Caputo derivative and R-L integral are formed:

$$
\begin{aligned}
& { }_{a}^{C} D_{t a}^{\alpha} J_{t}^{\alpha} u(t)=u(t) ; \quad a \leq t \leq b \\
& { }_{a} J_{t}^{\alpha}{ }_{a}^{C} D_{t}^{\alpha} u(t)=u(t)-\sum_{k=0}^{m-1} \frac{u^{(k)}(a)}{k !}(t-a)^{k}
\end{aligned}
$$

vi. Let $\alpha \geq 0 ; m=\lceil\alpha\rceil$ and for $u(t)=(t-a)^{\beta}$ for some $\beta \geq 0$. Then:

$$
{ }_{a}^{C} D_{t}^{\alpha} u(t)=\left\{\begin{array}{cl}
0 & \text { if } \beta \in\{0,1,2, \cdots, m-1\} \\
\frac{\Gamma(\beta+1)}{\Gamma(\beta+1-\alpha)}(t-a)^{\beta-\alpha} & \text { if } \beta \in \mathbb{N} \text { and } \beta \geq m \\
\text { or } \beta \notin \mathbb{N} \text { and } \beta>m-1
\end{array}\right.
$$

vii. The Laplace transform of the convolution of two functions is the product of their Laplace transforms.

$$
\mathcal{L}\{(u * v)(t), p\}=\mathcal{L}\left\{\int_{0}^{t} u(t-\eta) v(\eta) d \eta, p\right\}=U(p) V(p)
$$

Where $U(p)=\mathcal{L}\{u(t), p\}$ and $V(p)=\mathcal{L}\{v(t), p\}$.

viii. The Laplace transform of an integral term, for any $t>0$ can be formed:

$$
\mathcal{L}\left\{\int_{0}^{t} u(\xi) d \xi, p\right\}=\frac{1}{p} U(p)
$$

ix. The Laplace transform for power function multiple to a function for all $t>0$, can be formed:

$$
\mathcal{L}\left\{t^{n} u(t), p\right\}=(-1)^{n} \frac{d^{n}}{d p^{n}} U(p)
$$

x. The Laplace transform of Caputo fractional of order $\alpha$ and $m=\lceil\alpha\rceil$, can be obtained as follows:

\subsection{The Adomian Decomposition method: $[4,14,15]$}

$$
\begin{array}{r}
\mathcal{L}\left\{{ }_{0}^{C} D_{t}^{\alpha} u(t), p\right\}=p^{-(m-\alpha)}\left[p^{m} U(p)-\sum_{k=0}^{m-1} p^{m-k-1} u^{(k)}(0)\right] \\
=p^{\alpha} U(p)-\sum_{k=0}^{m-1} p^{\alpha-k-1} u^{(k)}(0)
\end{array}
$$

The Adomian decomposition method is one of the most powerful techniques used to solve semianalytically the nonlinear equations. In this method, decomposing the unknown function $u(t)$ of an equation into a sum of an infinite number of elements described through the decomposition series:

$$
u(t)=\sum_{r=0}^{\infty} u_{r}(t)
$$

Where the components $u_{r}(t), r \geq 0$ are to be determined in a recursive manner, this method concerns itself with finding the components $u_{0}(t), u_{1}(t), u_{2}(t), \cdots$ individually. However the nonlinear term $F(u(t))$, can be written in the decomposed form: 


$$
F(u(t))=\sum_{n=0}^{\infty} A_{n}\left[u_{0}(t), u_{1}(t), \cdots, u_{n}(t)\right]
$$

Where $A_{n}{ }^{\prime} s$ are called the Adomian polynomials which depending on $u_{0}, u_{1}, \cdots, u_{n}$ and that are obtained for the nonlinearity $F(u(t))$ by formula:

$$
A_{n}(t)=\frac{1}{n !} \frac{d^{n}}{d \theta^{n}}\left[F\left(\sum_{i=0}^{\infty} \theta^{i} u_{i}\right)\right]_{\theta=0}, n=0,1,2, \cdots
$$

Where $\theta$ is a parameter introduced for convenience. The uniqueness of the Adomian polynomial is not required at all and we can apply the Taylor expansion of $F(u(t))$ about the first component $u_{0}(t)$ to generate the forms as follows:

$$
F(u(t))=\sum_{i=0}^{\infty} A_{i}\left[u_{0}(t), u_{1}(t), \cdots, u_{i}(t)\right]=\sum_{i=0}^{\infty} \frac{\left[u(t)-u_{0}(t)\right]^{i}}{i !} F^{(i)}\left(u_{0}(t)\right)
$$

Through the use of above expansions, from the simple analytic nonlinearity $F(u(t))$, the Adomian polynomials $A_{n}{ }^{\prime} s$ are arranged to have the form:

$$
\begin{aligned}
& A_{0}=\frac{1}{0 !} \frac{d^{0}}{d \theta^{0}}\left[F\left(\sum_{i=0}^{0} \theta^{i} u_{i}\right)\right]_{\theta=0}=F\left(u_{0}\right) \\
& \begin{aligned}
& A_{1}=\frac{1}{1 !} \frac{d^{1}}{d \theta^{1}}\left[F\left(\sum_{i=0}^{1} \theta^{i} u_{i}\right)\right]_{\theta=0}=\frac{1}{1 !} \frac{d^{1}}{d \theta^{1}}\left[F\left(\theta^{0} u_{0}+\theta^{1} u_{1}\right)\right]_{\theta=0}=\left[F^{\prime}\left(\theta^{0} u_{0}+\theta^{1} u_{1}\right)\right]_{\theta=0}\left(u_{1}\right) \\
&=u_{1} F^{\prime}\left(u_{0}\right){ }^{2}=\frac{1}{2 !} \frac{d^{2}}{d \theta^{2}}[F\left.\left(\sum_{i=0}^{2} \theta^{i} u_{i}\right)\right]_{\theta=0}=\frac{1}{2 !} \frac{d^{2}}{d \theta^{2}}\left[F\left(\theta^{0} u_{0}+\theta^{1} u_{1}+\theta^{2} u_{2}\right)\right]_{\theta=0} \\
&= \frac{1}{2 !} \frac{d}{d \theta}\left[F^{\prime}\left(\theta^{0} u_{0}+\theta^{1} u_{1}+\theta^{2} u_{2}\right)\left(u_{1}+2 \theta u_{2}\right)\right]_{\theta=0} \\
&= \frac{1}{2 !}\left[F^{\prime}\left(\theta^{0} u_{0}+\theta^{1} u_{1}+\theta^{2} u_{2}\right)\left(2 u_{2}\right)\right. \\
&\left.+F^{\prime \prime}\left(\theta^{0} u_{0}+\theta^{1} u_{1}+\theta^{2} u_{2}\right)\left(u_{1}+2 \theta u_{2}\right)^{2}\right]_{\theta=0}=u_{2} F^{\prime}\left(u_{0}\right)+\frac{1}{2 !} u_{1}^{2} F^{\prime \prime}\left(u_{0}\right) \\
& A_{3}=u_{3} F^{\prime}\left(u_{0}\right)+u_{1} u_{2} F^{\prime \prime}\left(u_{0}\right)+\frac{1}{3 !} u_{1}^{3} F^{\prime \prime \prime}\left(u_{0}\right) \\
& A_{4}=u_{4} F^{\prime}\left(u_{0}\right)+\left(\frac{1}{2 !} u_{2}^{2}+u_{1} u_{3}\right) F^{\prime \prime}\left(u_{0}\right)+\frac{1}{2 !} u_{1}^{2} u_{2} F^{\prime \prime \prime}\left(u_{0}\right)+\frac{1}{4 !} u_{1}^{4} F^{(4)}\left(u_{0}\right)
\end{aligned}
\end{aligned}
$$

and so on. For this case the Adomian polynomial $A_{n}(t)=A_{n}\left[u_{0}(t), u_{1}(t), \cdots, u_{n}(t)\right], n \geq 1$, can be listed in general formula, [16]:

Where

$$
A_{n}(t)=\sum_{k=1}^{n} C_{n}^{k} F^{(k)}\left(u_{0}\right)
$$

$$
C_{n}^{k}=\left\{\begin{array}{lc}
u_{n}, & k=1 \\
\frac{1}{n} \sum_{j=0}^{n-k}(j+1) u_{j+1} C_{n-1-j}^{k-1}, & 2 \leq k \leq n
\end{array}\right.
$$

A useful tool that will speed up the convergence of the ADM is developed; the new technique relies upon in most cases the so-called "Noise Term Phenomenon" [13] that demonstrate a speedy convergence of the solution. The noise terms phenomenon can be used for all functional equations like the differential or integral equations. The noise terms are defined as the identical terms with opposite signs that can also appear in the components $u_{0}(t)$ and $u_{1}(t)$ and in the other components as well, Noise terms may appear if the exact solution of the equation is part of the zeroth component $u_{0}(t)$. Verification that the remaining non-canceled terms satisfy the integral equation is necessary and essential.

\section{Solution Technique for multi-higher IFDE of V-H type:}

In this section, we shall derive the semi-analytical solution of the nonlinear (IFDE) of the (V-H) type of the form (1) under the initial condition (2) by using Laplace transform that combine with the Adomian decomposition method and Modified Adomian decomposition method according to two different types of kernels difference and first order-simple degenerate kernels which can make a general formula of each ones: 


\subsection{Apply the LADM for Difference Kernel:}

Consider the nonlinear (IFDE) for difference kernel $\mathcal{K}_{\ell}(t, s)=\mathcal{K}_{\ell}(t-s)$ of the (V-H) type of the form (1) under the initial condition (2). Suppose $F_{\ell}(u(s))=\mathcal{H}_{\ell}\left(s,{ }_{0}^{C} D_{s}^{\beta_{\ell}} u(s)\right)$, for all $\ell=$ $0,1 \cdots, m$; and put $\mathcal{F}(p)$ as a Laplace transform of $f(t)$. First, apply the Laplace transform for both sides of equation (1) with the aid of Laplace transform of Caputo derivatives differentiation property as in equation (4) and using convolution property (vii) for difference kernel type, the following equation is obtained:

Where

$$
\begin{aligned}
& \psi(p) U(p)=\sum_{k=0}^{m_{\alpha_{n}}-1} p^{\alpha_{n}-k-1} u_{k}+\sum_{i=1}^{n-1} \mathcal{C}_{i}\left[\sum_{k=0}^{m_{\alpha_{i}}-1} p^{\alpha_{i}-k-1} u_{k}\right]+\mathcal{F}(p) \\
& +\sum_{\ell=0}^{m} \lambda_{\ell} \mathcal{L}\left\{\mathcal{K}_{\ell}(t)\right\} \mathcal{L}\left\{F_{\ell}(u(t))\right\}
\end{aligned}
$$

$$
\psi(p)=p^{\alpha_{n}}+\sum_{i=1}^{n-1} \mathcal{C}_{i} p^{\alpha_{i}}+\mathcal{C}_{0}
$$

Second, to overcome the difficulty of the nonlinear term $F_{\ell}(u(t))$, we apply the Adomian decomposition method for handling (5). To achieve this goal, we first represent the linear term $u(t)$ at the left side by an infinite series of components in the form:

$$
u(t)=\sum_{r=0}^{\infty} u_{r}(t)
$$

Where the components $u_{r}(t), r \in \mathbb{N}_{0}$ will be recursively determined, and obtained equation (7b) after applying the Laplace transform for both sides of equation (7a), with noted that for each $r$, $\mathcal{L}\left\{u_{r}(t), p\right\}=U_{r}(p)$ :

$$
U(p)=\sum_{r=0}^{\infty} U_{r}(p)
$$

However, the nonlinear term $F_{\ell}(u(t))$ at the right side (5) will be represented by an infinite series of the Adomian polynomials in the form, for all $\ell=0,1, \cdots, m$ :

$$
F_{\ell}(u(t))=\sum_{r=0}^{\infty} A_{r}^{\ell}\left[u_{0}(t), u_{1}(t), \cdots, u_{r}(t)\right]
$$

At last, substituting equations ( $7 \mathrm{~b}, 7 \mathrm{a}$ and 8 ) into (5) then leads to the following recursive relation:

Where

$$
\left.\begin{array}{c}
U_{0}(p)=\frac{1}{\psi(p)}\left\{\sum_{k=0}^{m_{\alpha_{n}}-1} p^{\alpha_{n}-k-1} u_{k}+\sum_{i=1}^{n-1} \mathcal{C}_{i}\left[\sum_{k=0}^{m_{\alpha_{i}}-1} p^{\alpha_{i}-k-1} u_{k}\right]+\mathcal{F}(p)\right\} \\
U_{k+1}(p)=\frac{1}{\psi(p)}\left\{\sum_{\ell=0}^{m} \lambda_{\ell} \mathcal{L}\left\{\mathcal{K}_{\ell}(t)\right\} \mathcal{L}\left\{A_{k}^{\ell}\left[u_{0}(t), u_{1}(t), \cdots, u_{k}(t)\right]\right\}\right\} ; \text { for } k \geq 0
\end{array}\right\}
$$

Applying the inverse Laplace transform to the first part of the equation (9) gives $u_{0}(t)=$ $\mathcal{L}^{-1}\left\{U_{0}(p), t\right\}$, so putting in the second part of equation (9) we obtain $U_{1}(p)$ and then getting $u_{1}(t)=$ $\mathcal{L}^{-1}\left\{U_{1}(p), t\right\}$. By same stages, this in turn will lead to the complete determination of the components of all $u_{k}(t) ; k \geq 0$.

In computational practice for the Adomian polynomials $A_{r}^{\ell}$, s we truncate the series after $r=M$ for positive finite number $M$. Thus: 


$$
A_{r}^{\ell}(t)=\frac{1}{r !} \frac{d^{r}}{d \theta^{r}}\left[F_{\ell}\left(\sum_{i=0}^{M} \theta^{i} u_{i}\right)\right]_{\theta=0}, 0 \leq r \leq M \text {; for all } \ell=0,1 \cdots, m
$$

So, all terms of the series in equation (7a) need not to be determined and so we use an approximation of the solution by the following truncated series:

$$
u(t) \cong \hat{u}_{M}(t)=\sum_{r=0}^{M} u_{r}(t), \quad M \in \mathbb{Z}^{+}
$$

The components $u_{0}, u_{1}, \cdots, u_{M}$ are determined recursively by the above formula (9) or using the noise terms approach idea. It is important to note that the decomposition method suggests that the zeroth component $u_{0}(t)$ be defined by the initial conditions and the function $f(t)$ as described above. The other components namely $u_{1}, u_{2}, \cdots, u_{M}$ are derived recurrently.

\subsection{Apply the LADM for Simple Degenerate Kernel:}

Laplace Adomian decomposition technique can be used to solve some kinds of nonlinear IFDE of $\mathrm{V}-\mathrm{H}$ type which the kernel is not necessarily differenced kernel, here we take the same equation (1) with all conditions (2) on that equation except the kernel which is a first order simple degenerate kernel: $\mathcal{K}_{\ell}(t, s)=c_{\ell} t+d_{\ell} s$, where $c_{\ell}$ and $d_{\ell}$ are any two positive-real numbers. Suppose that $F_{\ell}(u(s))=\mathcal{H}_{\ell}\left(s,{ }_{0}^{C} D_{s}^{\beta_{\ell}} u(s)\right)$; and put $\mathcal{F}(p)$ as a Laplace transform of $f(t)$. First, apply the Laplace transform for both sides of with the aid of fractional differentiation property (vii) of Laplace transform for Caputo derivatives, the following equation is obtained:

Where

$$
\begin{gathered}
U(p)=\mathcal{L}\{u(t)\}=\frac{1}{\psi(p)}\left\{\sum_{k=0}^{m_{\alpha_{n}-1}} p^{\alpha_{n}-k-1} u_{k}+\sum_{i=1}^{n-1} \mathcal{C}_{i}\left[\sum_{k=0}^{m_{\alpha_{i}-1}} p^{\alpha_{i}-k-1} u_{k}\right]+\mathcal{F}(p)\right\} \\
+\frac{1}{\psi(p)}\left\{\sum_{\ell=0}^{m} \lambda_{\ell}\left(c_{\ell} \mathcal{L}\left\{I_{t}^{\ell}\right\}+d_{\ell} \mathcal{L}\left\{I_{s}^{\ell}\right\}\right)\right\}
\end{gathered}
$$

$$
I_{t}^{\ell}=\int_{0}^{t} t F_{\ell}(u(s)) d s ; I_{s}^{\ell}=\int_{0}^{t} s F_{\ell}(u(s)) d s
$$

and $\psi(p)$ given in equation (6). Taking Laplace transforms for $I_{t}^{\ell}$ and $I_{s}^{\ell}, \ell=0,1, \cdots, m$; respectively, and calling properties (viii and ix) for integral parts, yields:

$$
\left.\begin{array}{l}
\mathcal{L}\left\{I_{t}^{\ell}\right\}=-\frac{d}{d p}\left(\frac{1}{p} \mathcal{L}\left\{F_{\ell}(u(s))\right\}\right) \\
\mathcal{L}\left\{I_{s}^{\ell}\right\}=-\frac{1}{p} \frac{d}{d p} \mathcal{L}\left\{F_{\ell}(u(s))\right\}
\end{array}\right\}
$$

Now, we represent the linear term $u(t)$ at the left side (12) by an infinite series of components defined in the equation (7a). However, the nonlinear term $F_{\ell}(u(s)) ; \ell=0,1, \cdots, m$ at the right side of it will be represented by an infinite series of the Adomian polynomials defined in the equation (8). Then substituting equations (16) and (7b) into (12) leads to the following recursive relation:

$$
\left.\begin{array}{r}
U_{0}(p)=\frac{1}{\psi(p)}\left\{\sum_{k=0}^{m_{\alpha_{n}}-1} p^{\alpha_{n}-k-1} u_{k}+\sum_{i=1}^{n-1} \mathcal{C}_{i}\left[\sum_{k=0}^{m_{\alpha_{i}}-1} p^{\alpha_{i}-k-1} u_{k}\right]+\mathcal{F}(p)\right\} \\
U_{k+1}(p)=\frac{-1}{\psi(p)}\left\{\sum _ { \ell = 0 } ^ { m } \lambda _ { \ell } \left[c_{\ell} \frac{d}{d p}\left(\frac{1}{p} \mathcal{L}\left\{A_{k}^{\ell}\left[u_{0}(t), u_{1}(t), \cdots, u_{k}(t)\right]\right\}\right)\right.\right. \\
\left.\left.\quad+d_{\ell} \frac{1}{p} \frac{d}{d p} \mathcal{L}\left\{A_{k}^{\ell}\left[u_{0}(t), u_{1}(t), \cdots, u_{k}(t)\right]\right\}\right]\right\} ; \text { for } k \geq 0
\end{array}\right\}
$$

Where $\psi(p)$ given in equation (6) and all $A_{k}^{\ell}\left[u_{0}(t), u_{1}(t), \cdots, u_{k}(t)\right]$ are defined in equation (10). Also, apply the inverse Laplace transform to the first part of the equation (14) gives $u_{0}(t)=$ $\mathcal{L}^{-1}\left\{U_{0}(p), t\right\}$, so putting in the second part of equation (14) we obtain $U_{1}(p)$ and then getting 
$u_{1}(t)=\mathcal{L}^{-1}\left\{U_{1}(p), t\right\}$. By the same stages, this, in turn, will lead to the complete determination of the components of all $u_{k}(t) ; 0 \leq k \leq M$. So we use an approximation of the solution by the truncated series (11).

\subsection{Apply the Modify LADM for Solving non-Linear IFDE of V-H type:}

The modified Adomian decomposition method with the aid of Laplace transform to solving nonlinear IFDE of V-H type of the form (1), introduces a slight variation to the recurrence relation (9) that will lead to the determination of the components of $u(t)$ in an easier and faster manner. For many cases, the function $f(t)$ in equation (1) can be set as the finite sum of partial functions $f_{i}(t), i=$ $0,1, \ldots, N(\in \mathbb{N})$ :

$$
f(t)=f_{0}(t)+f_{1}(t)+\cdots+f_{N}(t)
$$

In view of (15), we introduce a qualitative change in the formation of the recurrence relation (9). To minimize the size of calculations, we identify the zeros component $u_{0}(t)$ by one part of $f(t)$, namely $f_{0}(t)$. The other part of $f(t)$ can be added to the component $u_{1}(t)$, namely $f_{1}(t)$, among other terms, Continue this process $N$-time. The modify Adomian decomposition technique with the aid of Laplace transform introduces the modified recurrence relation for difference kernel type:

$$
\begin{gathered}
U_{0}(p)=\frac{1}{\psi(p)}\left\{\sum_{k=0}^{m_{\alpha_{n}}-1} p^{\alpha_{n}-k-1} u_{k}+\sum_{i=1}^{n-1} \mathcal{C}_{i}\left[\sum_{k=0}^{m_{\alpha_{i}}-1} p^{\alpha_{i}-k-1} u_{k}\right]+\mathcal{F}_{0}(p)\right\} \\
\left.U_{i}(p)=\frac{\mathcal{F}_{i}(p)}{\psi(p)}+\frac{1}{\psi(p)}\left\{\sum_{\ell=0}^{m} \lambda_{\ell} \mathcal{L}\left\{\mathcal{K}_{\ell}(t)\right\} \mathcal{L}\left\{A_{i-1}^{\ell}\left[u_{0}(t), u_{1}(t), \cdots, u_{i}(t)\right]\right\}\right\},(i \geq 1)\right\} \\
U_{k+1}(p)=\frac{1}{\psi(p)}\left\{\sum_{\ell=0}^{m} \lambda_{\ell} \mathcal{L}\left\{\mathcal{K}_{\ell}(t)\right\} \mathcal{L}\left\{A_{k}^{\ell}\left[u_{0}(t), u_{1}(t), \cdots, u_{k}(t)\right]\right\}\right\} ; \text { for } k \geq N
\end{gathered}
$$

Where $\mathcal{F}_{i}(p)$ is Laplace transform of $f_{i}(t)$. This shows that the difference between the standard recurrence relation (9) and the modified recurrence relation (16) rests only in the formation of the first $M$-components $u_{0}(t), u_{1}(t)$ and so on to $u_{M}(t)$. The other components $u_{j}, j>M$ remain the same in the third recurrence relations. Although this variation in the formation of $u_{0}(t), u_{1}(t), \ldots, u_{M}(t)$ is slight, however it plays a major role in accelerating the convergence of the solution and in minimizing the size of computational work.

Applying the inverse Laplace transform to the first part of equation (16) gives $u_{0}(t)=$ $\mathcal{L}^{-1}\left\{U_{0}(p), t\right\}$, so putting in the second part of equation (16) we obtain $U_{1}(p)$ and then getting $u_{1}(t)=\mathcal{L}^{-1}\left\{U_{1}(p), t\right\}$. By same stages, this in turn will lead to the complete determination of the components of all $u_{k}(t)$. So we use an approximation of the solution by the truncated series (11).

Moreover, Laplace transform with MADM can also be applied for first order simple degenerate kernels: $\mathcal{K}_{\ell}(t, s)=c_{\ell} t+d_{\ell} s$, where $c \& d \in \mathbb{R}^{+}$. Thus by the same procedure as in section 3.2 and from equations (12 and 13) we obtain the following recurrence relations respectively:

$$
\left.\begin{array}{c}
U_{0}(p)=\frac{1}{\psi(p)}\left\{\sum_{k=0}^{m_{\alpha_{n}}-1} p^{\alpha_{n}-k-1} u_{k}+\sum_{i=1}^{n-1} \mathcal{C}_{i}\left[\sum_{k=0}^{m_{\alpha_{i}}-1} p^{\alpha_{i}-k-1} u_{k}\right]+\mathcal{F}_{0}(p)\right\} \\
U_{i}(p)=\frac{\mathcal{F}_{i}(p)}{\psi(p)}-\frac{1}{\psi(p)} \sum_{\ell=0}^{m} \lambda_{\ell}\left[c_{\ell} \frac{d}{d p}\left(\frac{1}{p} \mathcal{L}\left\{A_{i-1}^{\ell}\left[u_{0}(t), u_{1}(t), \cdots, u_{i}(t)\right]\right\}\right)\right. \\
\left.\quad+d_{\ell} \frac{1}{p} \frac{d}{d p} \mathcal{L}\left\{A_{i-1}^{\ell}\left[u_{0}(t), u_{1}(t), \cdots, u_{i}(t)\right]\right\}\right], i \geq 1 \\
U_{k+1}(p)=\frac{-1}{\psi(p)}\left\{\sum _ { \ell = 0 } ^ { m } \lambda _ { \ell } \left[c_{\ell} \frac{d}{d p}\left(\frac{1}{p} \mathcal{L}\left\{A_{k}^{\ell}\left[u_{0}(t), u_{1}(t), \cdots, u_{k}(t)\right]\right\}\right)\right.\right. \\
\left.\left.+d_{\ell} \frac{1}{p} \frac{d}{d p} \mathcal{L}\left\{A_{k}^{\ell}\left[u_{0}(t), u_{1}(t), \cdots, u_{k}(t)\right]\right\}\right]\right\} ; \text { for } k \geq N
\end{array}\right\}
$$

\section{Illustrative Examples:}

In this section, we shall give some illustrative examples in order to clarify our approach. We consider the following test problems: 
Example 1: We first consider a higher-order nonlinear IFDE of V-H type with variable coefficients:

Where

$$
{ }_{0}^{c} D_{t}^{\alpha} u(t)=f(t)+\lambda \int_{0}^{t}(t-s)\left\{{ }_{0}^{C} D_{s}^{\beta} u(s)\right\}^{r} d s ; 0<\alpha<1
$$

$$
f(t)=\frac{6}{\Gamma(3-\alpha)} t^{2-\alpha}-\frac{\lambda 3^{r} \Gamma^{r}(3)}{(2 r-\beta r+1)(2 r-\beta r+2) \Gamma^{r}(3-\beta)} t^{2 r-\beta r+2} ; r \in \mathbb{N}
$$

And $\lambda \in \mathbb{R}$ subject to the initial condition $u(0)=0$.

Now, if we take $\beta=0$ and $r=1$ we get the linear IFDE. From here put $\alpha=0.5$ and $\lambda=\frac{1}{3}$ then from the equation above we have:

$$
\mathcal{K}_{0}(t, s)=(t-s) ; m_{\alpha}=1 ; \mathcal{L}\left\{\mathcal{K}_{0}(t), p\right\}=\frac{1}{p^{2}} \text { and } \mathcal{F}(p)=\frac{6}{p^{2.5}}-\frac{2}{p^{5}}
$$

and $F_{0}(u(s))={ }_{0}^{C} D_{s}^{0} u(s)=u(s)$. From equation (6) yields: $\psi(p)=p^{0.5}$

Using the first part of recursive relation (9) we obtain:

$$
U_{0}(p)=\mathcal{L}\left\{u_{0}(t), p\right\}=\frac{6}{p^{3}}-\frac{2}{p^{5.5}}
$$

Taking inverse Laplace to transform for the above equation yields

Using equation (10) where $\ell=0$, we get:

$$
u_{0}(t)=\mathcal{L}^{-1}\left\{U_{0}(p), t\right\}=3 t^{2}-\frac{2}{\Gamma(5.5)} t^{9 / 2}
$$

$A_{0}^{0}=F_{0}\left(u_{0}(t)\right)=3 t^{2}-\frac{2}{\Gamma(5.5)} t^{4.5}$ and $\mathcal{L}\left\{A_{0}^{0}(t), p\right\}=\frac{6}{p^{3}}-\frac{2}{p^{5.5}}$

Applying the second part of recursive relation (9) with $k=0$ we obtain:

$$
U_{1}(p)=\mathcal{L}\left\{u_{1}(t), p\right\}=\frac{2}{p^{5.5}}-\frac{2}{3 p^{8}}
$$

Taking inverse Laplace to transform for the above equation yields

From equation (10) to find $A_{1}^{0}$ put $\ell=0$, thus:

$$
u_{1}(t)=\frac{2}{\Gamma(5.5)} t^{9 / 2}-\frac{2}{3 \Gamma(8)} t^{7}
$$

and

$$
A_{1}^{0}=u_{1} F_{0}^{\prime}\left(u_{0}(t)\right)=u_{1} * 1=\frac{2}{\Gamma(5.5)} t^{4.5}-\frac{2}{3 \Gamma(8)} t^{7}
$$

$$
\mathcal{L}\left\{A_{1}^{0}(t), p\right\}=\frac{2}{p^{5.5}}-\frac{2}{3 p^{8}}
$$

Applying the second part of recursive relation (9) with $k=1$, we obtain:

$$
U_{2}(p)=\mathcal{L}\left\{u_{2}(t), p\right\}=\frac{2}{3 p^{8}}-\frac{2}{9 p^{10.5}}
$$

Taking inverse Laplace to transform for the above equation yields

Using equation (10), with $\ell=0$ we get:

$$
u_{2}(t)=\frac{2}{3 \Gamma(8)} t^{7}-\frac{2}{9 \Gamma(10.5)} t^{19 / 2}
$$

$$
\begin{gathered}
A_{2}^{0}=u_{2} F^{\prime}\left(u_{0}\right)+\frac{1}{2 !} u_{1}^{2} F^{\prime \prime}\left(u_{0}\right)=u_{2} * 1+0=\frac{2}{3 \Gamma(8)} t^{7}-\frac{2}{9 \Gamma(10.5)} t^{9.5} \\
\mathcal{L}\left\{A_{2}^{0}(t), p\right\}=\frac{2}{3 p^{8}}-\frac{2}{9 p^{10.5}}
\end{gathered}
$$

Applying the second part of recursive relation (9) with $k=2$ we obtain:

$$
U_{3}(p)=\mathcal{L}\left\{u_{3}(t), p\right\}=\frac{2}{9 p^{10.5}}-\frac{2}{27 p^{13}}
$$

Taking inverse Laplace to transform for the above equation yields

By the same technique for $k=3$ we get

$$
u_{3}(t)=\frac{2}{3^{2} \Gamma(10.5)} t^{19 / 2}-\frac{2}{3^{3} \Gamma(13)} t^{12}
$$

$$
u_{4}(t)=\frac{2}{3^{3} \Gamma(13)} t^{12}-\frac{2}{3^{4} \Gamma(15.5)} t^{29 / 2}
$$


In general $k \geq 1$ we get

$$
u_{k}(t)=\frac{2}{3^{k-1} \Gamma(3+5 \mathrm{k} / 2)} t^{(5 k+4) / 2}-\frac{2}{3^{k} \Gamma(11 / 2+5 \mathrm{k} / 2)} t^{(5 k+9) / 2}
$$

Considering (11), the approximated solution for $k=1,3$ and 5 are:

$$
\begin{aligned}
& u(t) \cong \hat{u}_{1}(t)=\sum_{i=0}^{1} u_{i}(t)=3 t^{2}-\frac{2}{3 \Gamma(8)} t^{7} \\
& u(t) \cong \hat{u}_{3}(t)=\sum_{i=0}^{3} u_{i}(t)=3 t^{2}-\frac{2}{3^{3} \Gamma(13)} t^{12} \\
& u(t) \cong \hat{u}_{5}(t)=\sum_{i=0}^{5} u_{i}(t)=3 t^{2}-\frac{2}{3^{5} \Gamma(18)} t^{17}
\end{aligned}
$$

The following table presents a comparison between the exact solution and the approximate solution $\hat{u}_{1}(t), \hat{u}_{3}(t)$ and $\hat{u}_{5}(t)$ respectively, depending on the least square error.

\begin{tabular}{|c|c|c|c|c|}
\hline \multirow{2}{*}{$\boldsymbol{t}$} & \multirow{2}{*}{ Exact solution } & \multicolumn{3}{|c|}{ Approximate Solutions } \\
\cline { 3 - 5 } & & $\hat{u}_{1}(t)$ & $\hat{u}_{3}(t)$ & $\hat{u}_{5}(t)$ \\
\hline \hline 0.0 & 0.0 & 0.0 & 0.0 & 0.0 \\
\hline 0.1 & 0.03 & 0.029999999986 & 0.03 & 0.03 \\
\hline 0.2 & 0.12 & 0.119999998306 & 0.12 & 0.12 \\
\hline 0.3 & 0.27 & 0.269999971071 & 0.27 & 0.27 \\
\hline 0.4 & 0.48 & 0.479999783280 & 0.48 & 0.48 \\
\hline 0.5 & 0.75 & 0.749998966600 & 0.75 & 0.75 \\
\hline 0.6 & 1.08 & 1.079996297142 & 1.079999999999 & 1.08 \\
\hline 0.7 & 1.47 & 1.469989106574 & 1.469999999997 & 1.47 \\
\hline 0.8 & 1.92 & 1.919972259894 & 1.919999999989 & 1.92 \\
\hline 0.9 & 2.43 & 2.429936733214 & 2.429999999956 & 2.43 \\
\hline 1.0 & 3.0 & 2.999867724867 & 2.999999999845 & 3.0 \\
\hline \hline & L.S. $\boldsymbol{E}$ & $2.24024 e-008$ & $2.59395 e-020$ & 0.0 \\
\hline
\end{tabular}

If we take $\beta=0.5$ and $r=2$ we get the nonlinear IFDE of V-H type, put $\alpha=0.5$ and $\lambda=\frac{1}{3}$ then from the basic problem we have:

Where

$$
{ }_{0}^{C} D_{t}^{0.5} u(t)=f(t)+\frac{1}{3} \int_{0}^{t}(t-s)\left[{ }_{0}^{C} D_{s}^{0.5} u(s)\right]^{2} d s
$$

$$
f(t)=\frac{6}{\Gamma(2.5)} t^{3 / 2}-\frac{3 \Gamma^{2}(3)}{20 \Gamma^{2}(2.5)} t^{5}
$$

Since we have the same kernel $\mathcal{K}_{0}(t, s)=t-s ; \mathcal{L}\left\{\mathcal{K}_{0}(t), p\right\}=\frac{1}{p^{2}}$ and $\mathcal{F}(p)=\frac{6}{p^{2.5}}-\frac{72}{\Gamma^{2}(2.5) p^{6}}$ and $F_{0}(u(s))=\left[{ }_{0}^{C} D_{s}^{0.5} u(s)\right]^{2}$. From equation (6) $\psi(p)=p^{0.5}$.Using the first part of recursive relation (9) we obtain:

Taking inverse Laplace to transform for the above equation yields

$$
U_{0}(p)=\mathcal{L}\left\{u_{0}(t), p\right\}=\frac{6}{p^{3}}-\frac{72}{\Gamma^{2}(2.5) p^{6.5}}
$$

$$
u_{0}(t)=\mathcal{L}^{-1}\left\{U_{0}(p), t\right\}=3 t^{2}-\frac{72}{\Gamma^{2}(2.5) \Gamma(6.5)} t^{5.5}
$$

To find $A_{0}^{0}$, from equation (10) putting $\ell=0$ and using the Caputo definition for order 0.5 we obtain:

$$
A_{0}^{0}(t)=A_{0}^{0}\left[u_{0}(t)\right]=F_{0}\left(u_{0}(t)\right)=\left[{ }_{0}^{C} D_{t}^{0.5} u_{0}(t)\right]^{2}=\frac{36}{\Gamma^{2}(2.5)} t^{3}-\frac{36}{5 \Gamma^{3}(2.5)} t^{6.5}+\frac{9}{25 \Gamma^{4}(2.5)} t^{10}
$$

Apply Laplace definition on $A_{0}^{0}(t)$, we get 


$$
\mathcal{L}\left\{A_{0}^{0}(t), p\right\}=\frac{216}{\Gamma^{2}(2.5) p^{4}}-\frac{36}{5 \Gamma^{3}(2.5) p^{7.5}}+\frac{9 \Gamma(11)}{25 \Gamma^{4}(2.5) p^{11}}
$$

Applying the second part of recursive relation (9) with $k=0$ we obtain:

$$
U_{1}(p)=\mathcal{L}\left\{u_{1}(t), p\right\}=\frac{72}{\Gamma^{2}(2.5) p^{6.5}}-\frac{12 \Gamma(7.5)}{5 \Gamma^{3}(2.5) p^{10}}+\frac{3 \Gamma(11)}{25 \Gamma^{4}(2.5) p^{13.5}}
$$

Taking inverse Laplace transform for the equation, yields

$$
u_{1}(t)=\mathcal{L}^{-1}\left\{U_{1}(p), t\right\}=\frac{72}{\Gamma^{2}(2.5) \Gamma(6.5)} t^{5.5}-\frac{12 \Gamma(7.5)}{5 \Gamma^{3}(2.5) \Gamma(10)} t^{9}+\frac{3 \Gamma(11)}{25 \Gamma^{4}(2.5) \Gamma(13.5)} t^{12.5}
$$

Thus the approximate solution by the truncated series using two iterate $u_{0}(t)$ and $u_{1}(t)$ :

$$
u(t) \cong \hat{u}_{1}(t)=u_{0}(t)+u_{1}(t)=3 t^{2}-\frac{12 \Gamma(7.5)}{5 \Gamma^{3}(2.5) \Gamma(10)} t^{9}+\frac{3 \Gamma(11)}{25 \Gamma^{4}(2.5) \Gamma(13.5)} t^{12.5}
$$

From equation (10) to find $A_{1}^{0}$ put $\ell=0$, thus:

$$
\begin{aligned}
A_{1}^{0}\left[u_{0}(t), u_{1}(t)\right] & =\frac{d}{d \theta}\left[F_{0}\left(u_{0}(t)+\theta u_{1}(t)\right)\right]_{\theta=0}=\left.\frac{d}{d \theta}\left[{ }_{0}^{C} D_{t}^{0.5}\left(u_{0}(t)+\theta u_{1}(t)\right)\right]^{2}\right|_{\theta=0} \\
& =2\left[{ }_{0}^{C} D_{t}^{0.5} u_{0}(t)\right]\left[{ }_{0}^{C} D_{t}^{0.5} u_{1}(t)\right] \\
& =\frac{36}{5 \Gamma^{3}(2.5)} t^{6.5}-\frac{144 \Gamma(7.5)}{5 \Gamma^{4}(2.5) \Gamma(9.5)} t^{10}+\frac{36 \Gamma(11)}{25 \Gamma^{5}(2.5) \Gamma(13)} t^{13.5}-\frac{18}{25 \Gamma^{4}(2.5)} t^{10} \\
& +\frac{72 \Gamma(7.5)}{25 \Gamma^{5}(2.5) \Gamma(9.5)} t^{13.5}-\frac{18 \Gamma(11)}{125 \Gamma^{6}(2.5) \Gamma(13)}
\end{aligned}
$$

After finding the Laplace transform for $A_{1}^{0}\left[u_{0}(t), u_{1}(t)\right]$ and putting the answer in the equation (9) we obtain $U_{2}(p)=\mathcal{L}\left\{u_{2}(t), p\right\}$, finally using the inverse Laplace operator yields:

$$
\begin{aligned}
u_{2}(t)=\mathcal{L}^{-1}\left\{U_{2}(p), t\right\} & \\
& =\frac{12 \Gamma(7.5)}{5 \Gamma^{3}(2.5) \Gamma(10)} t^{9}-\frac{3 \Gamma(11)}{5 \Gamma^{4}(2.5) \Gamma(13.5)}\left[\frac{16 \Gamma(7.5)}{\Gamma(9.5)}+\frac{2}{5}\right] t^{12.5} \\
& +\frac{12 \Gamma(14.5)}{25 \Gamma^{5}(2.5) \Gamma(17)}\left[\frac{\Gamma(11)}{\Gamma(13)}+\frac{2 \Gamma(7.5)}{\Gamma(9.5)}\right] t^{16}-\frac{6 \Gamma(11) \Gamma(18)}{125 \Gamma^{6}(2.5) \Gamma(13) \Gamma(20.5)} t^{19.5}
\end{aligned}
$$

Thus the approximate solution by the truncated series using three iterate $u_{0}(t), u_{1}(t)$ and $u_{2}(t)$ :

$$
\begin{aligned}
u(t) \cong \hat{u}_{2}(t)= & u_{0}(t)+u_{1}(t)+u_{2}(t) \\
& =3 t^{2}-\frac{3 \Gamma(11)}{5 \Gamma^{4}(2.5) \Gamma(13.5)}\left[\frac{16 \Gamma(7.5)}{\Gamma(9.5)}+\frac{1}{5}\right] t^{12.5} \\
& +\frac{12 \Gamma(14.5)}{25 \Gamma^{5}(2.5) \Gamma(17)}\left[\frac{\Gamma(11)}{\Gamma(13)}+\frac{2 \Gamma(7.5)}{\Gamma(9.5)}\right] t^{16}-\frac{6 \Gamma(11) \Gamma(18)}{125 \Gamma^{6}(2.5) \Gamma(13) \Gamma(20.5)} t^{19.5}
\end{aligned}
$$

By the same procedure, we can find $u_{3}(t)$ and putting in approximate form then we obtain $\hat{u}_{3}(t)$ :

$$
\begin{aligned}
u(t) \cong \hat{u}_{3}(t)= & \sum_{i=0}^{3} u_{i}(t) \\
& =3 t^{2}-\frac{12 \Gamma(14.5)}{5 \Gamma^{5}(2.5) \Gamma(17)}\left[\frac{\Gamma(11)}{5 \Gamma(13)}+\frac{2 \Gamma(7.5)}{5 \Gamma(9.5)}+\frac{16 \Gamma(11) \Gamma(7.5)}{\Gamma(13) \Gamma(9.5)}\right] t^{16} \\
& +\frac{6 \Gamma(18)}{25 \Gamma^{6}(2.5) \Gamma(20.5)}\left[\frac{16 \Gamma(11) \Gamma(7.5)}{\Gamma(13) \Gamma(9.5)}+\frac{2 \Gamma(11)}{5 \Gamma(13)}+\frac{8 \Gamma(11) \Gamma(14.5)}{\Gamma(13) \Gamma(16.5)}+\frac{16 \Gamma(14.5) \Gamma(7.5)}{\Gamma(16.5) \Gamma(9.5)}\right. \\
& \left.+\frac{8 \Gamma^{2}(7.5)}{\Gamma^{2}(9.5)}\right] t^{19.5} \\
& -\frac{24 \Gamma(21.5)}{125 \Gamma^{7}(2.5) \Gamma(23.5)}\left[\frac{\Gamma(11) \Gamma(14.5)}{\Gamma(13) \Gamma(16.5)}+\frac{2 \Gamma(7.5) \Gamma(14.5)}{\Gamma(9.5) \Gamma(16.5)}+\frac{\Gamma(11) \Gamma(18)}{\Gamma(13) \Gamma(20)}+\frac{\Gamma(11) \Gamma(7.5)}{\Gamma(13) \Gamma(9.5)}\right] t^{22.5} \\
& +\frac{3 \Gamma(11) \Gamma(25)}{625 \Gamma^{8}(2.5) \Gamma(13) \Gamma(27.5)}\left[\frac{\Gamma(11)}{\Gamma(13)}-\frac{4 \Gamma(18)}{\Gamma(20)}\right] t^{26.5}
\end{aligned}
$$


The following table presents a comparison between the exact solution and the approximate solution $\hat{u}_{1}(t), \hat{u}_{2}(t)$ and $\hat{u}_{3}(t)$ respectively, depending on the least square error.

\begin{tabular}{|c|c|c|c|c|}
\hline \multirow{2}{*}{$\boldsymbol{t}$} & \multirow{2}{*}{ Exact solution } & \multicolumn{3}{|c|}{ Approximate Solutions } \\
\cline { 3 - 5 } & & $\hat{u}_{1}(t)$ & $\hat{u}_{2}(t)$ & $\hat{u}_{3}(t)$ \\
\hline 0.0 & 0.0 & 0.0 & 0.0 & 0.0 \\
\hline 0.1 & 0.03 & 0.02999999999 & 0.03 & 0.03 \\
\hline 0.2 & 0.12 & 0.1199999973 & 0.12 & 0.12 \\
\hline 0.3 & 0.27 & 0.2699998963 & 0.2699999999 & 0.27 \\
\hline 0.4 & 0.48 & 0.4799986198 & 0.4799999981 & 0.48 \\
\hline 0.5 & 0.75 & 0.7499897244 & 0.7499999683 & 0.7499999999 \\
\hline 0.6 & 1.08 & 1.079947045 & 1.079999691 & 1.079999998 \\
\hline 0.7 & 1.47 & 1.469788348 & 1.469997888 & 1.46999998 \\
\hline 0.8 & 1.92 & 1.919297908 & 1.919988841 & 1.919999829 \\
\hline 0.9 & 2.43 & 2.427980786 & 2.429951663 & 2.429998883 \\
\hline 1.0 & 3.0 & 2.994813197 & 2.999821107 & 2.999994036 \\
\hline \hline & L.S. $\boldsymbol{2}$ & \multirow{2}{*}{$3.15208 e-05$} & \multirow{2}{*}{$3.4469 e-08$} & \multirow{2}{*}{$3.684 e-11$} \\
\hline
\end{tabular}

Example 2: Consider a higher-order nonlinear IFDE of V-H type:

Where

$$
{ }_{0}^{C} D_{t}^{\alpha} u(t)=f(t)+\int_{0}^{t}(t-s)^{2}\left\{{ }_{0}^{c} D_{s}^{\beta_{1}} u(s)\right\}^{2} d s+\int_{0}^{t}(t-s)\left\{{ }_{0}^{C} D_{s}^{\beta_{2}} u(s)\right\}^{3} d s
$$

$$
\begin{gathered}
f(t)=\frac{2}{\Gamma(3-\alpha)} t^{2-\alpha}-\frac{4}{\Gamma^{2}\left(3-\beta_{1}\right)}\left\{\frac{t^{7-2 \beta_{1}}}{5-2 \beta_{1}}-\frac{2 t^{7-2 \beta_{1}}}{6-2 \beta_{1}}+\frac{t^{7-2 \beta_{1}}}{7-2 \beta_{1}}\right\} \\
-\frac{8}{\Gamma^{3}\left(3-\beta_{2}\right)}\left\{\frac{t^{8-3 \beta_{2}}}{7-3 \beta_{2}}-\frac{t^{8-3 \beta_{2}}}{8-3 \beta_{2}}\right\}
\end{gathered}
$$

And $1<\alpha, \beta_{1}, \beta_{2}<2$ with the initial condition $u(0)=-9, u^{\prime}(0)=2$.

Now, if we take $\alpha=1.1, \beta_{1}=1.5, \beta_{2}=1.8$ then from the equation above we have:

$$
\begin{gathered}
\mathcal{K}_{2}(t, s)=(t-s) ; \mathcal{K}_{1}(t, s)=(t-s)^{2} ; \mathcal{K}_{0}(t, s)=0 ; m_{\alpha}=2 \\
\mathcal{L}\left\{\mathcal{K}_{2}(t)\right\}=\frac{1}{p^{2}} ; \mathcal{L}\left\{\mathcal{K}_{1}(t)\right\}=\frac{2}{p^{3}} ; \mathcal{F}(p)=\frac{2}{p^{1.9}}-\frac{8}{\Gamma^{2}(1.5) p^{5}}-\frac{25 \Gamma(3.6)}{13 \Gamma^{3}(1.2) p^{3.6}}
\end{gathered}
$$

Let $\quad F_{2}(u(s))=\left[{ }_{0}^{C} D_{s}^{1.8} u(s)\right]^{3} ; \quad F_{1}(u(s))=\left[{ }_{0}^{C} D_{s}^{1.5} u(s)\right]^{2}$

From equation (6) yields: $\psi(p)=p^{1.1}$ Using the first part of recursive relation (9) we obtain:

$$
U_{0}(p)=\mathcal{L}\left\{u_{0}(t)\right\}=\frac{2}{p^{2}}-\frac{9}{p}+\frac{2}{p^{3}}-\frac{8}{\Gamma^{2}(1.5) p^{6.1}}-\frac{25 \Gamma(3.6)}{13 \Gamma^{3}(1.2) p^{4.7}}
$$

Taking inverse Laplace to transform for the above equation yields

$$
u_{0}(t)=t^{2}+2 t-9-\frac{8}{\Gamma^{2}(1.5) \Gamma(6.1)} t^{5.1}-\frac{25 \Gamma(3.6)}{13 \Gamma^{3}(1.2) \Gamma(4.7)} t^{3.7}
$$

Using equation (10), with $\ell=1,2$ we get:

$$
\begin{aligned}
& A_{0}^{1}=F_{1}\left(u_{0}\right)=\left(\frac{2}{\Gamma(1.5)} t^{0.5}-\frac{8}{\Gamma^{2}(1.5) \Gamma(4.6)} t^{3.6}-\frac{25 \Gamma(3.6)}{13 \Gamma^{3}(1.2) \Gamma(3.2)} t^{2.2}\right)^{2} \\
& A_{0}^{2}=F_{2}\left(u_{0}\right)=\left(\frac{2}{\Gamma(1.2)} t^{0.2}-\frac{8}{\Gamma^{2}(1.5) \Gamma(4.3)} t^{3.3}-\frac{25 \Gamma(3.6)}{13 \Gamma^{3}(1.2) \Gamma(2.9)} t^{1.9}\right)^{3}
\end{aligned}
$$

Applying the second part of recursive relation (9) with $k=0$ we obtain:

$$
U_{1}(p)=\mathcal{L}\left\{u_{1}(t)\right\}=\frac{8}{\Gamma^{2}(1.5) p^{6.1}}+\frac{8 \Gamma(1.6)}{\Gamma^{3}(1.2) p^{4.7}}+\cdots
$$

Taking inverse Laplace to transform for the above equation yields

$$
u_{1}(t)=\frac{8}{\Gamma^{2}(1.5) \Gamma(6.1)} t^{5.1}+\frac{8 \Gamma(1.6)}{\Gamma^{3}(1.2) \Gamma(4.7)} t^{3.7}+\cdots
$$


The noise terms $\pm \frac{8}{\Gamma^{2}(1.5) \Gamma(6.1)} t^{5.1}$ and $\pm \frac{8 \Gamma(1.6)}{\Gamma^{3}(1.2) \Gamma(4.7)} t^{3.7}$ appears in $u_{0}(t)$ and $u_{1}(t)$. Cancelling these terms from the zeros component $u_{0}(t)$ gives the solution which is the exact solution:

$$
u(t)=t^{2}+2 t-9
$$

Example 3: Consider the following nonlinear IFDE of V-H type

where

$$
{ }_{0}^{C} D_{t}^{1.6} u(t)=f(t)+\int_{0}^{t}(t-s)^{2 C} D_{s}^{1.2} u(s) d s-\int_{0}^{t}(t-s)\left\{{ }_{0}^{C} D_{s}^{1.7} u(s)\right\}^{2} d s
$$

With the initial condition $u(0)=7, u^{\prime}(0)=-3$

$$
f(t)=\frac{4}{\Gamma(1.4)} t^{0.4}-\frac{500}{1197 \Gamma(1.8)} t^{3.8}+\frac{50}{13 \Gamma^{2}(1.3)} t^{2.6}
$$

While the exact solution is $u(t)=7-3 t+2 t^{2}$.

Now, from the problem we have:

$$
\begin{gathered}
\mathcal{K}_{2}(t, s)=-(t-s) ; \mathcal{K}_{1}(t, s)=(t-s)^{2} ; \mathcal{K}_{0}(t, s)=0 \\
\alpha=1.6 ; \beta_{2}=1.7, \quad \beta_{1}=1.2, \beta_{0}=0 ; m_{\alpha}=2 \\
F_{2}(u(s))=\left\{{ }_{0}^{C} D_{s}^{1.7} u(s)\right\}^{2} ; \quad F_{1}(u(s))={ }_{0}^{C} D_{s}^{1.2} u(s)
\end{gathered}
$$

Apply standard LADM: Using the first part of recursive relation (9) to find $U_{0}(p)$ where $u_{0}=$ $7 ; u_{1}=-3$ and From equation (6), for $\alpha_{1}=1.6$ and all $\mathcal{C}_{i}$ 's are zeros, we obtain $\psi(p)=p^{1.6}$. Then taking the inverse Laplace to transform for both sides we get:

$$
u_{0}(t)=\mathcal{L}^{-1}\left\{U_{0}(p), t\right\}=7-3 t+2 t^{2}-\frac{8}{\Gamma(6.4)} t^{5.4}+\frac{50 \Gamma(3.6)}{13 \Gamma^{2}(1.3) \Gamma(5.2)} t^{4.2}
$$

To find $A_{0}^{1}$ and $A_{0}^{2}$, from equation (10) putting $\ell=0,1$ and using the Caputo definition for order 1.2 and 1.7 with $F_{0}$ and $F_{1}$, respectively, we obtain:

$$
\begin{gathered}
A_{0}^{1}(t)=A_{0}^{1}\left[u_{0}(t)\right]=F_{0}\left(u_{0}(t)\right)={ }_{0}^{C} D_{t}^{1.2} u_{0}(t)=\frac{4}{\Gamma(1.8)} t^{0.8}-\frac{8}{\Gamma(5.2)} t^{4.2}+\frac{50 \Gamma(3.6)}{\left.13 \Gamma^{2}(1.3) \Gamma\right)(4)} t^{3} \\
A_{0}^{2}(t)=A_{0}^{1}\left[u_{0}(t)\right]=F_{1}\left(u_{0}(t)\right)=\left[{ }_{0}^{C} D_{t}^{1.7} u_{0}(t)\right]^{2} \\
=\frac{16}{\Gamma^{2}(1.3)} t^{0.6}-\frac{64}{\Gamma(1.3) \Gamma(4.7)} t^{4}+\frac{400 \Gamma(3.6)}{13 \Gamma^{3}(1.3) \Gamma(3.5)} t^{2.8}+\frac{64}{\Gamma^{2}(4.7)} t^{7.4} \\
-\frac{800 \Gamma(3.6)}{13 \Gamma^{2}(1.3) \Gamma(4.7) \Gamma(3.5)} t^{6.2}+\frac{2500 \Gamma^{2}(3.6)}{169 \Gamma^{4}(1.3) \Gamma^{2}(3.5)} t^{5}
\end{gathered}
$$

Apply Laplace definition on $A_{0}^{1}(t), A_{0}^{2}(t)$, and the Laplace transform of each kernel is $\mathcal{L}\left\{\mathcal{K}_{2}(t)\right\}=$ $\frac{-1}{p^{2}} ; \mathcal{L}\left\{\mathcal{K}_{1}(t)\right\}=\frac{2}{p^{3}}$ with using the formula (9) we obtain $U_{1}(p)$. Finally take Laplace inverse for both sides we get the $u_{1}(t)$ :

$$
\begin{aligned}
u_{1}(t)=\mathcal{L}^{-1}\left\{U_{1}(p), t\right\} & \frac{8}{\Gamma(6.4)} t^{5.4}-\frac{16}{\Gamma(9.8)} t^{8.8}+\frac{4}{\Gamma(1.3) \Gamma(8.6)}\left[\frac{25 \Gamma(3.6)}{13 \Gamma(1.3)}+\frac{16 \Gamma(5)}{\Gamma(4.7)}\right] t^{7.6} \\
& -\frac{16 \Gamma(1.6)}{\Gamma^{2}(1.3) \Gamma(5.2)} t^{4.2}-\frac{400 \Gamma(3.6) \Gamma(3.8)}{13 \Gamma^{3}(1.3) \Gamma(3.5) \Gamma(7.4)} t^{6.4}-\frac{64 \Gamma(8.4)}{\Gamma^{2}(4.7) \Gamma(12)} t^{11} \\
& +\frac{800 \Gamma(3.6) \Gamma(7.2)}{13 \Gamma^{2}(1.3) \Gamma(4.7) \Gamma(3.5) \Gamma(10.8)} t^{9.8}-\frac{2500 \Gamma^{2}(3.6) \Gamma(6)}{169 \Gamma^{4}(1.3) \Gamma^{2}(3.5) \Gamma(9.6)} t^{8.6}
\end{aligned}
$$

Thus the approximate solution by the truncated series using two iterate $u_{0}(t)$ and $u_{1}(t)$ :

$$
\begin{aligned}
u(t) \cong \hat{u}_{1}(t)= & u_{0}(t)+u_{1}(t) \\
& =7-3 t+2 t^{2}-\frac{16}{\Gamma(9.8)} t^{8.8}+\frac{4}{\Gamma(1.3) \Gamma(8.6)}\left[\frac{25 \Gamma(3.6)}{13 \Gamma(1.3)}+\frac{16 \Gamma(5)}{\Gamma(4.7)}\right] t^{7.6} \\
& -\frac{400 \Gamma(3.6) \Gamma(3.8)}{13 \Gamma^{3}(1.3) \Gamma(3.5) \Gamma(7.4)} t^{6.4}-\frac{64 \Gamma(8.4)}{\Gamma^{2}(4.7) \Gamma(12)} t^{11} \\
& +\frac{800 \Gamma(3.6) \Gamma(7.2)}{13 \Gamma^{2}(1.3) \Gamma(4.7) \Gamma(3.5) \Gamma(10.8)} t^{9.8}-\frac{2500 \Gamma^{2}(3.6) \Gamma(6)}{169 \Gamma^{4}(1.3) \Gamma^{2}(3.5) \Gamma(9.6)} t^{8.6}
\end{aligned}
$$

Putting $k=1$ from the second part of equation (9) and using the Laplace transforms of kernels $\mathcal{L}\left\{\mathcal{K}_{1}(t)\right\}$ and $\mathcal{L}\left\{\mathcal{K}_{2}(t)\right\}$ with valued od $\lambda_{\ell}$ 's in the equation, yields 


$$
U_{2}(p)=\frac{1}{p^{1.6}}\left\{\frac{2}{p^{3}} \mathcal{L}\left\{A_{1}^{1}\left[u_{0}(t), u_{1}(t)\right]\right\}-\frac{1}{p^{2}} \mathcal{L}\left\{A_{1}^{2}\left[u_{0}(t), u_{1}(t)\right]\right\}\right\}
$$

After finding each of $A_{1}^{1}$ and $A_{1}^{2}$ by using defining the $F_{1}$ and $F_{2}$, respectively and determine Laplace transforms with putting all into the formula above. Finally, taking the inverse Laplace transform to obtain $u_{2}(t)$. Thus the approximate solution by the truncated series using three iterations $u_{0}(t), u_{1}(t)$ and $u_{2}(t)$ :

$$
\begin{aligned}
u(t) \cong \hat{u}_{1}(t)= & u_{0}(t)+u_{1}(t)+u_{2}(t) \\
& =7-3 t+2 t^{2}-\frac{32}{\Gamma(13.2)} t^{12.2} \\
& +\left\{\frac{8}{\Gamma(1.3) \Gamma(12)}\left[\frac{25 \Gamma(3.6)}{13 \Gamma(1.3)}+\frac{16 \Gamma(5)}{\Gamma(4.7)}\right]+\frac{64 \Gamma(8.4)}{\Gamma(12)}\left[\frac{2}{\Gamma(1.3) \Gamma(8.1)}+\frac{1}{\Gamma^{2}(4.7)}\right]\right\} t^{11} \\
& +\frac{100 \Gamma(3.6) \Gamma(6)}{13 \Gamma^{4}(1.3) \Gamma(3.5) \Gamma(9.6)}\left[\frac{32 \Gamma(3.8)}{\Gamma(5.7)}-\frac{24 \Gamma(1.6)}{\Gamma(3.5)}\right] t^{8.6} \\
& -\frac{1}{\Gamma(10.8)}\left\{\frac{256 \Gamma(1.6)}{\Gamma^{2}(1.3) \Gamma(3.5)}\left[\frac{\Gamma(3.8)}{\Gamma(1.3)}-\frac{\Gamma(7.2)}{\Gamma(4.7)}\right]+\frac{32 \Gamma(7.2)}{\Gamma^{2}(1.3) \Gamma(6.9)}\left[\frac{25 \Gamma(3.6)}{13 \Gamma(1.3)}+\frac{16 \Gamma(5)}{\Gamma(4.7)}\right]\right\} t^{9.8} \\
& -\frac{128}{\Gamma(4.7) \Gamma(15.4)}\left[\frac{\Gamma(8.4)}{\Gamma(4.7)}+\frac{2 \Gamma(11.8)}{\Gamma(8.1)}\right] t^{14.4}-\frac{1024 \Gamma(8.4) \Gamma(14)}{\Gamma^{3}(4.7) \Gamma(10.8) \Gamma(17.6)} t^{16.6} \\
& +\frac{64}{\Gamma(1.3) \Gamma(14.2)}\left\{\frac{25 \Gamma(3.6)}{13 \Gamma(1.3) \Gamma(3.5)}\left[\frac{\Gamma(7.2)}{\Gamma(4.7)}+\frac{\Gamma(10.6)}{\Gamma(8.1)}\right]\right. \\
& \left.+\frac{\Gamma(10.6)}{\Gamma(4.7)}\left[\frac{8 \Gamma(8.4)}{\Gamma(4.7) \Gamma(10.3)}+\frac{25 \Gamma(3.6)}{13 \Gamma(1.3) \Gamma(6.9)}+\frac{16 \Gamma(5)}{\Gamma(4.7) \Gamma(6.9)}\right]\right\} t^{13.2} \\
& -\frac{200 \Gamma(3.6)}{13 \Gamma^{3}(1.3) \Gamma(3.5) \Gamma(13)}\left\{\frac{25 \Gamma(3.6)}{13 \Gamma(1.3)}\left[\frac{\Gamma(6)}{\Gamma(3.5)}+\frac{2 \Gamma(9.4)}{\Gamma(6.9)}\right]\right. \\
& \left.+\frac{32 \Gamma(9.4)}{\Gamma(4.7)}\left[\frac{\Gamma(7.2)}{\Gamma(9.1)}+\frac{\Gamma(3.8)}{\Gamma(5.7)}+\frac{\Gamma(5)}{\Gamma(6.9)}\right]\right\} t^{12} \\
& +\frac{20000 \Gamma^{2}(3.6) \Gamma(8.2)}{169 \Gamma^{5}(1.3) \Gamma^{2}(3.5) \Gamma(11.8)}\left[\frac{\Gamma(6)}{\Gamma(7.9)}+\frac{2 \Gamma(3.8)}{\Gamma(5.7)}\right] t^{10.8} \\
& +\frac{6400 \Gamma(3.6) \Gamma(12.8)}{13 \Gamma^{2}(1.3) \Gamma^{2}(4.7) \Gamma(3.5) \Gamma(16.4)}\left[\frac{\Gamma(8.4)}{\Gamma(10.3)}+\frac{2 \Gamma(7.2)}{\Gamma(9.1)}\right] t^{15.4} \\
& -\frac{40000 \Gamma^{2}(3.6) \Gamma(11.6)}{169 \Gamma^{4}(1.3) \Gamma^{2}(3.5) \Gamma(4.7) \Gamma(15.2)}\left[\frac{\Gamma(6)}{\Gamma(7.9)}+\frac{2 \Gamma(7.2)}{\Gamma(9.1)}\right] t^{14.2} \\
& +\frac{250000 \Gamma^{3}(3.6) \Gamma(6) \Gamma(10.4)}{2197 \Gamma^{6}(1.3) \Gamma^{3}(3.5) \Gamma(7.9) \Gamma(14)} t^{13}
\end{aligned}
$$

The following table presents a comparison between the exact solution and the approximate solution $\hat{u}_{0}(t), \hat{u}_{1}(t)$ and $\hat{u}_{2}(t)$ respectively, depending on the least square error.

\begin{tabular}{|c|c|c|c|c|}
\hline \multirow{2}{*}{$\boldsymbol{t}$} & \multirow{2}{*}{ Exact solution } & \multicolumn{3}{|c|}{ Approximate Solutions } \\
\cline { 3 - 5 } & 7.0 & $\hat{u}_{0}(t)$ & $\hat{u}_{1}(t)$ & $\hat{u}_{2}(t)$ \\
\hline 0.0 & 6.72 & 7.0 & 7.0 & 7.0 \\
\hline 0.1 & 6.48 & 6.720034244 & 6.719999942 & 6.72 \\
\hline 0.2 & 6.28 & 6.283418818 & 6.479995145 & 6.479999955 \\
\hline 0.3 & 6.12 & 6.131376244 & 6.119587953 & 6.279998544 \\
\hline 0.4 & 6.0 & 6.028856817 & 5.998270101 & 6.119983051 \\
\hline 0.5 & 5.92 & 5.981645921 & 5.914391509 & 5.999887496 \\
\hline 0.6 & 5.88 & 5.996965041 & 5.864774937 & 5.878116676 \\
\hline 0.7 & 5.88 & 6.083463905 & 5.843683769 & 5.874363761 \\
\hline 0.8 & 5.92 & 6.251199649 & 5.841486751 & 5.905445168 \\
\hline 0.9 & 6.0 & 6.511605461 & 5.842873373 & 5.966721596 \\
\hline 1.0 & L.S.E & 0.431286 & 0.032438 & 0.001354 \\
\hline \hline
\end{tabular}


Apply Modified LADM: We first split $f(t)$ into two parts $(N=1)$, namely:

Then the Laplace transform became:

$$
f_{0}(t)=\frac{4}{\Gamma(1.4)} t^{0.4} \text { and } f_{1}(t)=\frac{-500}{1197 \Gamma(1.8)} t^{3.8}+\frac{50}{13 \Gamma^{2}(1.3)} t^{2.6}
$$

$$
\mathcal{F}_{0}(p)=\frac{4}{p^{1.4}} \quad \text { and } \quad \mathcal{F}_{1}(p)=\frac{-500 \Gamma(4.8)}{1197 \Gamma(1.8) p^{4.8}}+\frac{50 \Gamma(3.6)}{13 \Gamma^{2}(1.3) p^{3.6}}
$$

Using the first part of recursive relation (16) to find $U_{0}(p)$ where $u_{0}=7 ; u_{1}=-3$ and From equation (6), for $\alpha_{1}=1.6$ and all $\mathcal{C}_{i}$ 's are zeros, we obtain $\psi(p)=p^{1.6}$, we obtain:

$$
U_{0}(p)=\mathcal{L}\left\{u_{0}(t)\right\}=\frac{-3}{p^{2}}+\frac{7}{p}+\frac{4}{p^{3}}
$$

Taking inverse Laplace to transform for the above equation yields

Using equation (10), with $\ell=1,2$ we get:

$$
u_{0}(t)=2 t^{2}-3 t+7
$$

$$
A_{0}^{1}\left[u_{0}(t)\right]=F_{1}\left(u_{0}(t)\right)=\frac{4}{\Gamma(1.8)} t^{0.8} ; A_{0}^{2}\left[u_{0}(t)\right]=F_{2}\left(u_{0}(t)\right)=\frac{16}{\Gamma^{2}(1.3)} t^{0.6}
$$

Applying the second part of recursive relation (16) we obtain:

$$
U_{1}(p)=\mathcal{L}\left\{u_{1}(t)\right\}=\frac{-500 \Gamma(4.8)}{1197 \Gamma(1.8) p^{6.4}}+\frac{50 \Gamma(3.6)}{13 \Gamma^{2}(1.3) p^{5.2}}+\frac{8}{p^{6.4}}-\frac{16 \Gamma(1.6)}{\Gamma^{2}(1.3) p^{5.2}}=0
$$

The Laplace inverse of the zero function is zero, so $u_{1}(t)=0$

Using the third part of recursive relation (16) we obtain, for $k \geq 1$ :

$$
U_{k+1}(p)=\mathcal{L}\left\{u_{k+1}(t)\right\}=\frac{1}{p^{1.6}}\left\{\sum_{\ell=0}^{2} \lambda_{\ell} \mathcal{L}\left\{\mathcal{K}_{\ell}(t)\right\} \mathcal{L}\left\{A_{k}^{\ell}\left[u_{0}(t), u_{1}(t), \cdots, u_{k}(t)\right]\right\}\right\}=0
$$

It is obvious that each component of $u_{j}, j \geq 1$ is zero. The solution is: $u(t)=2 t^{2}-3 t+7$. which is the exact solution to our problem.

Example 4: Consider the following nonlinear IFDE of V-H type

where

$$
{ }_{0}^{C} D_{t}^{0.6} u(t)=f(t)+\int_{0}^{t}(t+s){ }_{0}^{C} D_{s}^{0.4} u(s)-(t+2 s)\left[{ }_{0}^{C} D_{s}^{0.5} u(s)\right]^{2} d s
$$

with the initial condition $u(0)=-7$.

$$
f(t)=\frac{2}{\Gamma(1.4)} t^{0.4}-\frac{42}{5 \Gamma(3.6)} t^{2.6}+\frac{14}{3 \Gamma^{2}(1.5)} t^{3}
$$

Now, from the problem we have: $\alpha=1.6 ; m_{\alpha}=2$ and

$$
\begin{aligned}
{\left[\begin{array}{lll}
\beta_{0} & \beta_{1} & \beta_{2}
\end{array}\right]=\left[\begin{array}{lll}
0 & 0.4 & 0.5
\end{array}\right] ;\left[\begin{array}{lll}
\lambda_{0} & \lambda_{1} & \lambda_{2}
\end{array}\right]=\left[\begin{array}{lll}
0 & 1 & -1
\end{array}\right] } \\
\mathcal{K}_{2}(t, s)=t+2 s ; \mathcal{K}_{1}(t, s)=t+s ; \mathcal{K}_{0}(t, s)=0 \\
F_{2}(u(s))=\left[\begin{array}{ll}
{ }_{0}^{C} D_{s}^{0.5} u(s)
\end{array}\right] \quad ; \quad F_{1}(u(s))={ }_{0}^{C} D_{s}^{0.4} u(s)
\end{aligned}
$$

The Laplace transform of the function $f(t)$ can formed as:

$$
\mathcal{F}(p)=\frac{2}{p^{1.4}}-\frac{42}{5 p^{3.6}}+\frac{28}{\Gamma^{2}(1.5) p^{4}}
$$

Apply standard LADM: Using the first part of recursive relation (16) to find $U_{0}(p)$ where $u_{0}=-7$; and From equation (6), for $\alpha=0.6$ and all $\mathcal{C}_{i}$ 's are zeros, we obtain $\psi(p)=p^{0.6}$. Then taking the inverse Laplace to transform for both sides we get:

$$
u_{0}(t)=\mathcal{L}^{-1}\left\{U_{0}(p), t\right\}=-7+2 t-\frac{42}{5 \Gamma(4.2)} t^{3.2}+\frac{28}{\Gamma^{2}(1.5) \Gamma(4.6)} t^{3.6}
$$

To find $A_{0}^{1}$ and $A_{0}^{2}$, from equation (10) putting $\ell=0,1$ and using the Caputo definition for order 0.4 and 0.5 with $F_{1}$ and $F_{2}$, respectively, we obtain:

$$
A_{0}^{1}(t)=A_{0}^{1}\left[u_{0}(t)\right]=F_{1}\left(u_{0}(t)\right)={ }^{C} D_{t}^{0.4} u_{0}(t)=\frac{2}{\Gamma(1.6)} t^{0.6}-\frac{42}{5 \Gamma(3.8)} t^{2.8}+\frac{28}{\left.\Gamma^{2}(1.5) \Gamma\right)(4.2)} t^{3.2}
$$




$$
\begin{aligned}
A_{0}^{2}(t)=A_{0}^{1}\left[u_{0}(t)\right] & =F_{2}\left(u_{0}(t)\right)=\left[{ }^{C} D_{t}^{0.5} u_{0}(t)\right]^{2} \\
& =\frac{4}{\Gamma^{2}(1.5)} t-\frac{168}{5 \Gamma(3.7) \Gamma(1.5)} t^{3.2}+\frac{112}{\Gamma^{3}(1.5) \Gamma(4.1)} t^{4.6}+\frac{1764}{25 \Gamma^{2}(3.7)} t^{5.4} \\
& -\frac{2352}{5 \Gamma^{2}(1.5) \Gamma(4.1) \Gamma(3.7)} t^{6.8}+\frac{784}{\Gamma^{4}(1.5) \Gamma^{2}(4.1)} t^{8.2}
\end{aligned}
$$

Apply Laplace definition on $A_{0}^{1}(t), A_{0}^{2}(t)$, and using the formula (16) we obtain $U_{1}(p)$, for $k=0$. Finally, take Laplace inverse for both sides we get the $u_{1}(t)$ :

$$
u_{1}(t)=\mathcal{L}^{-1}\left\{U_{1}(p), t\right\}
$$

$$
\begin{aligned}
& =\frac{42}{5 \Gamma(4.2)} t^{3.2}-\frac{1806}{25 \Gamma(6.4)} t^{5.4}+\frac{1316}{5 \Gamma^{2}(1.5) \Gamma(6.8)} t^{5.8}-\frac{28}{\Gamma^{2}(1.5) \Gamma(4.6)} t^{3.6} \\
& +\frac{11424 \Gamma(4.2)}{25 \Gamma(1.5) \Gamma(3.7) \Gamma(6.8)} t^{5.8}-\frac{9968 \Gamma(5.6)}{5 \Gamma^{3}(1.5) \Gamma(4.1) \Gamma(8.2)} t^{7.2}-\frac{22805 \Gamma(6.4)}{16 \Gamma^{2}(3.7) \Gamma(9)} t^{8} \\
& +\frac{45911 \Gamma(7.8)}{4 \Gamma^{2}(1.5) \Gamma(4.1) \Gamma(3.7) \Gamma(10.4)} t^{9.4}-\frac{112112 \Gamma(9.2)}{5 \Gamma^{4}(1.5) \Gamma^{2}(4.1) \Gamma(11.8)} t^{10.8}
\end{aligned}
$$

The noise terms $\pm \frac{42}{5 \Gamma(4.2)} t^{3.2}$ and $\pm \frac{28}{\Gamma^{2}(1.5) \Gamma(4.6)} t^{3.6}$ appears in $u_{0}(t)$ and $u_{1}(t)$. Cancelling these terms from the zeros component $u_{0}(t)$, as the theorem of Noise terms in iterative, then gives the solution which is the exact solution: $u(t)=2 t-7$.

Apply Modified LADM: We first split $f(t)$ into two parts $(N=1)$, namely:

Then the Laplace transform became:

$$
f_{0}(t)=\frac{2}{\Gamma(1.4)} t^{0.4} \text { and } f_{1}(t)=-\frac{42}{5 \Gamma(3.6)} t^{2.6}+\frac{14}{3 \Gamma^{2}(1.5)} t^{3}
$$

$$
\mathcal{F}_{0}(p)=\frac{2}{p^{1.4}} \quad \text { and } \quad \mathcal{F}_{1}(p)=-\frac{42}{5 p^{3.6}}+\frac{28}{\Gamma^{2}(1.5) p^{4}}
$$

Using the first part recursive relation (17) we obtain: $U_{0}(p)=\mathcal{L}\left\{u_{0}(t)\right\}=-\frac{7}{p}+\frac{2}{p^{2}}$.

Taking inverse Laplace to transform for above equation yields $u_{0}(t)=-7+2 t$. Applying equation (10), with $\ell=1,2$ we get:

$$
A_{0}^{1}(t)=F_{1}\left(u_{0}(t)\right)=\frac{2}{\Gamma(1.6)} t^{0.6} ; A_{0}^{2}(t)=F_{2}\left(u_{0}(t)\right)=\frac{4}{\Gamma^{2}(1.5)} t
$$

Applying the second part of recursive relation (17) yields

$$
U_{1}(p)=\mathcal{L}\left\{u_{1}(t)\right\}=\frac{-8.4}{p^{4.2}}-\frac{20}{\Gamma^{2}(1.5) p^{4.6}}+\frac{8.4}{p^{4.2}}+\frac{20}{\Gamma^{2}(1.5) p^{4.6}}=0
$$

So $u_{1}(t)=0$. Then from the third part of recurrence relation (17), it follows immediately that $u_{k}(t)=0, \forall k \geq 2$. So the exact solution $u(t)=2 t-7$, readily obtain.

\section{Conclusion}

In this paper, Adomian and modified Adomian decomposition method has been successfully applied to finding the approximate as well as an exact solution of nonlinear integro-fractional differential equations of the Volterra-Hammerstein type. These techniques are very powerful and efficient in finding analytical as well as a numerical solution to our problem. It provides more realistic series solution that converges very rapidly to the solutions.

A considerable advantage of the method is that if we do not obtain the exact solution, then the solution can be written as a form of truncated series, and then $u(t)$ can be easily evaluated for arbitrary values of $t$. To obtain the best approximation we must use more terms. Sometimes the noise terms in Adomian method will not appear, so we use modified Adomian decomposition method.

\section{References}

1. Mittal, R. C. and Nigam R. 2008. Solution of Fractional Integro-Differential Equations by Adomian Decomposition Method. Int. J. of Appl. Math. and Mech. 4(42): 87-94.

2. Golberg, A.M. 1979. Solution methods for integral equations: theory and applications. New York: plenum press.

3. Jerri, A.J. 1971. Introduction to integral equations with applications. New York: Marcel Dekker.

4. Wazwaz, A.M. 2011. Linear and Nonlinear Integral Equations, Methods and Application. Springer Heidelberg Dordrecht London New York. 
5. Wazwaz, A.M and Mehanna M. S. 2010. The Combined Laplace-Adomian Method for Handling Singular Integral Equation of Heat Transfer. International Journal of Nonlinear Science, Vol. 10, No.2, pp248-252.

6. Arife, A.S., Korashe, S.T. and Yildirim, A. 2011. Laplace Adomian Decomposition Method for Solving a Model Chronic Myelogenous Leukemia CML and T Cell Interaction. World Applied Sciences Journal 13 (4): 756-761.

7. Manafianheris, J. 2012. Solving the Integro-Differential Equations Using the Modified Laplace Adomian Decomposition Method. Journal of Mathematical Extension Vol. 6, No. 1, 41-55.

8. Zurigat, M. 2012. Annals of the University of Craiova, Mathematics and Computer Science Series; Volume 39(2), Pages 200-210.

9. Kilbas, A. A., Srivastava, H. M. and Trujillo J. J. 2006. Theory and Applications of Fractional Differential Equations. Elsevier B.V. Netherlands.

10. Podldubny, I. 1999. Fractional Differential Equation. Academic press, San Diego.

11. Hilfer, R. 2000. Applications of Fractional Calculus in Physics, World Scientific.

12. Odibat, Z. M. and shawagfeh, N. T. 2007. Generalized Taylor's Formula, Applied Mathematics and computation, Vol.186 pp. 286-293.

13. Atanackovic, T. M., Pilipovic, S., Stankovic, B. and Zorica, D. 2014. Fractional Calculus with Applications in Mechanics; John Wiley and Sons, Inc.

14. Al-awawdah, E. 2016. The Adomian Decomposition Method for Solving Partial Differential Equations. M.Sc. Thesis Birzeit University.

15. Adomian, G. 1994. Solving Frontier Problems of Physics: The Decomposition Method. Kluwer Academic.

16. Duan, J.S. 2011. Convenient analytic recurrence algorithms for the Adomian polynomials, Appl. Math. Comput. 217, 6337-6348. 